\title{
Transcriptomic and phylogenetic analysis of Culex pipiens quinquefasciatus for three detoxification gene families
}

\author{
Liangzhen Yan ${ }^{1,2}$, Pengcheng Yang ${ }^{1}$, Feng Jiang ${ }^{1}, \mathrm{Na}$ Cui ${ }^{1}$, Enbo $\mathrm{Ma}^{2}$, Chuanling Qiao ${ }^{1}$ and Feng Cui ${ }^{1 *}$
}

\begin{abstract}
Background: The genomes of three major mosquito vectors of human diseases, Anopheles gambiae, Aedes aegypti, and Culex pipiens quinquefasciatus, have been previously sequenced. C. p. quinquefasciatus has the largest number of predicted protein-coding genes, which partially results from the expansion of three detoxification gene families: cytochrome P450 monooxygenases (P450), glutathione S-transferases (GST), and carboxyl/cholinesterases (CCE). However, unlike An. gambiae and Ae. aegypti, which have large amounts of gene expression data, C. p. quinquefasciatus has limited transcriptomic resources. Knowledge of complete gene expression information is very important for the exploration of the functions of genes involved in specific biological processes. In the present study, the three detoxification gene families of C. p. quinquefasciatus were analyzed for phylogenetic classification and compared with those of three other dipteran insects. Gene expression during various developmental stages and the differential expression responsible for parathion resistance were profiled using the digital gene expression (DGE) technique.
\end{abstract}

Results: A total of 302 detoxification genes were found in C. p. quinquefasciatus, including 71 CCE, 196 P450, and 35 cytosolic GST genes. Compared with three other dipteran species, gene expansion in Culex mainly occurred in the CCE and P450 families, where the genes of a-esterases, juvenile hormone esterases, and CYP325 of the CYP4 subfamily showed the most pronounced expansion on the genome. For the five DGE libraries, 3.5-3.8 million raw tags were generated and mapped to 13314 reference genes. Among 302 detoxification genes, 225 (75\%) were detected for expression in at least one DGE library. One fourth of the CCE and P450 genes were detected uniquely in one stage, indicating potential developmentally regulated expression. A total of 1511 genes showed different expression levels between a parathion-resistant and a susceptible strain. Fifteen detoxification genes, including 2 CCEs, 6 GSTs, and 7 P450s, were expressed at higher levels in the resistant strain.

Conclusions: The results of the present study provide new insights into the functions and evolution of three detoxification gene families in mosquitoes and comprehensive transcriptomic resources for C. p. quinquefasciatus, which will facilitate the elucidation of molecular mechanisms underlying the different biological characteristics of the three major mosquito vectors.

Keywords: Carboxyl/cholinesterases, Cytochrome P450 monooxygenases, Glutathione S-transferases, Insecticide resistance, Gene expansion, Gene expression

\footnotetext{
* Correspondence: cuif@ioz.ac.cn

${ }^{1}$ State Key Laboratory of Integrated Management of Pest Insects \& Rodents, Institute of Zoology, Chinese Academy of Sciences, Beijing 100101, China Full list of author information is available at the end of the article
} 


\section{Background}

Mosquitoes are the most important vectors of human diseases. The Culex pipiens complex has a broad geographic distribution and is the vector of the West Nile virus and the Wuchereria bancrofti nematode, which causes filariasis. Over the last several decades, chemical insecticides have been intensively applied to control disease transmission. However, such control is undermined seriously by the increased insecticide resistance of vector mosquitoes. Three gene families are implicated in insecticide metabolism in mosquitoes: cytochrome P450 monooxygenases (P450s) are responsible for pyrethroid resistance [1], glutathione S-transferases (GSTs) are responsible for DDT resistance [2], and carboxyl/cholinesterases (CCEs) are responsible for organophosphate and carbamate resistance [3]. Many insect species show rapid expansion and diversification of detoxification genes, as disclosed by their sequenced genomes. The expansion or restriction of detoxification genes likely helps insects adapt to their particular ecological niches and enable them to survive natural and man-made insecticide selection.

The genomes of three major taxonomic mosquitoes, including Anopheles gambiae, Aedes aegypti, and Culex pipiens quinquefasciatus, have been analyzed and released to the public [4-6]. Of the three, Ae. aegypti has the largest genome size (1376 Mb), while C. p. quinquefasciatus has the largest number of predicted proteincoding genes (18883), which is $22 \%$ larger than that of Ae. aegypti and $52 \%$ larger than that of An. gambiae. The extra number of protein-coding genes partially results from the expansion of its three detoxification gene families. However, unlike An. gambiae and Ae. aegypti, which have large amounts of gene expression data, such as various expressed sequence tag libraries and transcriptomes, C. p. quinquefasciatus has limited gene expression resources, with only several salivary gland transcriptomes currently reported $[7,8]$. Knowledge of complete gene expression information is very important for the exploration of the functions of genes involved in specific biological processes and for the discovery of new candidate genes.

In the present study, the three detoxification gene families of $C$. p. quinquefasciatus were subjected to phylogenetic analysis and compared with those of three other dipteran insects. The CCE and P450 families were found to undergo large gene expansion. Digital gene expression tag profiling (DGE) technology was used to perform a deep transcriptome analysis of C. p. quinquefasciatus during development and in response to organophosphate insecticide selection. The gene expression profiles obtained provide an invaluable resource for the identification of genes involved in the development and insecticide resistance of C. p. quinquefasciatus.

\section{Results and discussion}

\section{C. $p$. quinquefasciatus detoxification gene families}

When $1 \mathrm{e}^{-10}$ was used in the HMMER searches, 79, 203, and 17 candidate genes of CCEs, P450s, and GSTs were identified in the C. p. quinquefasciatus genome, respectively. After verified by community annotations, only 71 CCEs and 196 P450s were confirmed. The eight false positives for CCEs were lipases or conserved hypothetical proteins and the seven false positives for P450s were groucho protein, 25-hydroxyvitamin D-1 alpha hydroxylase, or conserved hypothetical proteins. When the search stringency was lessened to $2 \mathrm{e}^{-2}, 35$ cytosolic GSTs were identified and supported by community annotations. Thus, a total of 302 detoxification genes were found in C. p. quinquefasciatus, including 71 CCE, 196 $\mathrm{P} 450$, and 35 GST genes, representing the widest gene expansion among the dipteran insect genomes sequenced thus far (Table 1). Gene expansion was mainly observed in the CCE and P450 families compared with three other dipteran species.

Seventy-one CCE sequences were detected in the C. $p$. quinquefasciatus genome, which was approximately $39 \%$ and 29\% gene-expanded compared with An. gambiae and Ae aegypti, respectively, and 2-fold the number of CCEs found in D. melanogaster (Table 1, Additional file 1). The new functional assignment proposed by Oakeshott et al. [9] was used to designate the clades in the CCE phylogeny (Figure 1). A total of 11 clades, representing dietary/detoxification, hormone/semiochemical processing, and neuro/developmental functions, were obtained. The numbers of C. p. quinquefasciatus CCEs in the three functional classes were 30,26 , and 15 , respectively (Table 1).

The number of CCEs in the neuro/developmental class was relatively conserved among the four dipteran insects. Similar conservation occurs in hymenopteran (Nasonia vitripennis, A. mellifera) and coleopteran (Tribolium castaneum) genomes [9], which reflects the relatively ancient origins of this class, where all members are catalytically inactive except for the acetylcholinesterases.

CCEs in dietary/detoxification and hormone/semiochemical processing classes expanded on the C. p. quinquefasciatus genome compared with the three other dipteran insects. Expansion mainly occurred in $\alpha$ esterases (30 genes in clade B) and juvenile hormone esterases (22 genes in clades F and G). For $\alpha$-esterases Culex showed rapid radiation in two clusters, which contained six and seven members, respectively, and Aedes displayed an obvious expansion in one cluster with five $\alpha$-esterases (Figure 2). The $\alpha$-esterases are thought to be involved in the development of metabolic resistance to insecticides; some examples include $\alpha \mathrm{E} 7$ of Lucilia cuprina [10] and esterase A (CpipI_CPIJ013918) 
Table 1 Classification of detoxification gene families in Drosophila melanogaster, Anopheles gambiae, Aedes aegypti, and Culex pipiens quinquefasciatus

\begin{tabular}{|c|c|c|c|c|}
\hline & D. melanogaster & A. gambiae & A. aegypti & C. p. quinquefasciatus \\
\hline \multicolumn{5}{|l|}{ CCE } \\
\hline \multicolumn{5}{|l|}{ Dietary/detoxification $^{*}$} \\
\hline B class (a-esterases) & 13 & 16 & 22 & $30(18)$ \\
\hline \multicolumn{5}{|l|}{ Hormone/semiochemical processing } \\
\hline D class (integument esterases) & 3 & 0 & 0 & $1(0)$ \\
\hline E class ( $\beta$-esterases) & 2 & 4 & 2 & $3(3)$ \\
\hline F class (dipteran JH esterases) & 3 & 6 & 7 & $13(5)$ \\
\hline G class (lepidopteran JH esterases) & 0 & 4 & 6 & $9(6)$ \\
\hline \multicolumn{5}{|l|}{ Neuro/developmental } \\
\hline $\mathrm{H}$ class (glutactins) & 5 & 10 & 7 & $6(5)$ \\
\hline I class (unknown) & 1 & 1 & 1 & $1(1)$ \\
\hline J class (acetylcholinesterases) & 1 & 2 & 2 & $2(2)$ \\
\hline K class (gliotactins) & 1 & 1 & 1 & $1(1)$ \\
\hline L class (neuroligins) & 4 & 5 & 5 & $3(3)$ \\
\hline M class (neurotactins) & 2 & 2 & 2 & $2(2)$ \\
\hline Total & 35 & 51 & 55 & $71(46)$ \\
\hline \multicolumn{5}{|l|}{ P450 } \\
\hline CYP2 & 6 & 10 & 11 & $14(12)$ \\
\hline CYP3 (include CYP6 and CYP9) & 36 & 42 & 84 & $88(72)$ \\
\hline CYP4 & 32 & 45 & 59 & $83(58)$ \\
\hline Mitochondrial & 11 & 9 & 10 & $11(8)$ \\
\hline Total & 85 & 106 & 164 & $196(150)$ \\
\hline \multicolumn{5}{|l|}{ GST } \\
\hline Delta & 11 & 12 & 8 & $14(13)$ \\
\hline Epsilon & 14 & 8 & 8 & $10(6)$ \\
\hline Omega & 5 & 1 & 1 & $1(1)$ \\
\hline Sigma & 1 & 1 & 1 & $1(1)$ \\
\hline Theta & 4 & 2 & 4 & $6(6)$ \\
\hline Zeta & 2 & 1 & 1 & $0(0)$ \\
\hline Others & 0 & 3 & 3 & $3(2)$ \\
\hline Total & 37 & 28 & 26 & 35 (29) \\
\hline
\end{tabular}

*The dietary/detoxification functional group follows the new system proposed by Oakeshott et al. (2010).

Data of D. melanogaster, An. gambiae, and Ae. aegypti are taken from Oakeshott et al. (2010).

The number of $C$. $p$. quinquefasciatus genes in brackets is from the DGE libraries.

CCE, carboxyl/cholinesterases; P450, cytochrome P450 monooxygenases; GST, glutathione S-transferases.

and esterase B (CpipJ_CPIJ013917) of C. p. quinquefasciatus [3]. Unlike the rapid radiation of other $\alpha$ esterases, the esterase $A$ and esterase $B$ were well conserved: secure 1:1:1 orthologs were found across Culex, Anopheles and Aedes (Figure 2). Twenty-eight other $\alpha$-esterases of the species are probably involved in the metabolism of endotoxins or naturally occurring dietary constituents. The $\alpha$ - and $\beta$-based nomenclatures are applied extensively to esterase isozymes in Drosophila according to their preferential hydrolysis of isomeric artificial substracts, $\alpha$ - and $\beta$-naphthyl acetate, respectively
[11]. The nomenclatures in themselves represent no broad biological distinctions. In the culicines, two esterase genes involved in organophosphate resistance are commonly designated esterase A and esterase B based on their ability to use preferentially $\alpha$ - or $\beta$-naphthyl acetate in the presence of equal quantities of both substrates $[3,12]$.

The large expansion of juvenile hormone esterases (JHEs) on the C. p. quinquefasciatus genome is interesting: 22 compared with 3 to 13 in three other dipteran genomes and 2 in the hymenopteran and coleopteran genomes [9]. When compared among the three 


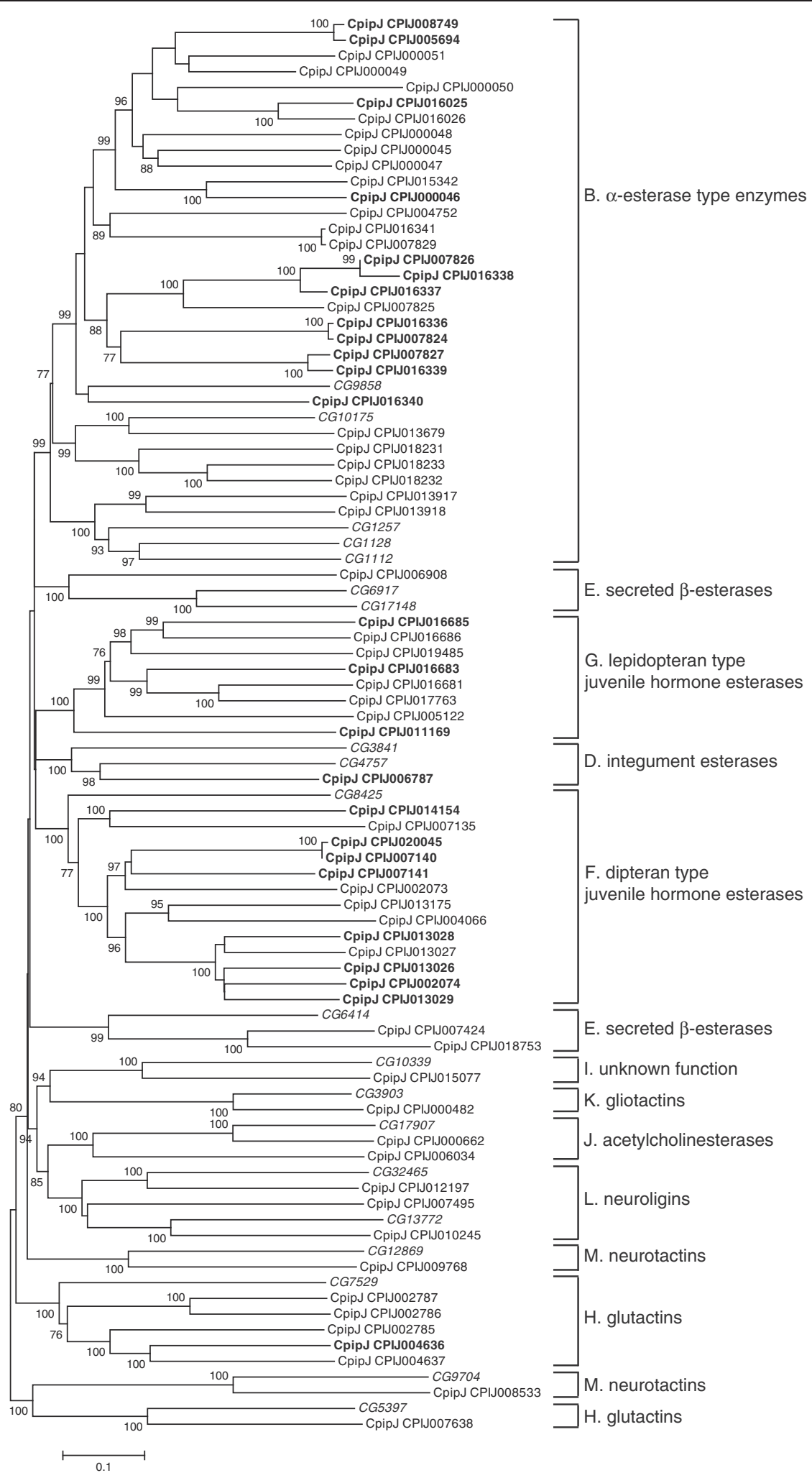

Figure 1 Unrooted distance neighbor-joining tree showing the phylogeny of carboxyl/cholinesterases (CCEs) from the genome of Culex pipiens quinquefasciatus in relation to CCEs from Drosophila melanogaster (in italics). CCEs of C. p. quinquefasciatus undetected in DGE libraries are shown in bold. The percentage of bootstrap confidence values greater than 70\% (1000 replicates) is shown at the nodes. The functional assignment of clades follows the new system proposed by Oakeshott et al. [9]. 


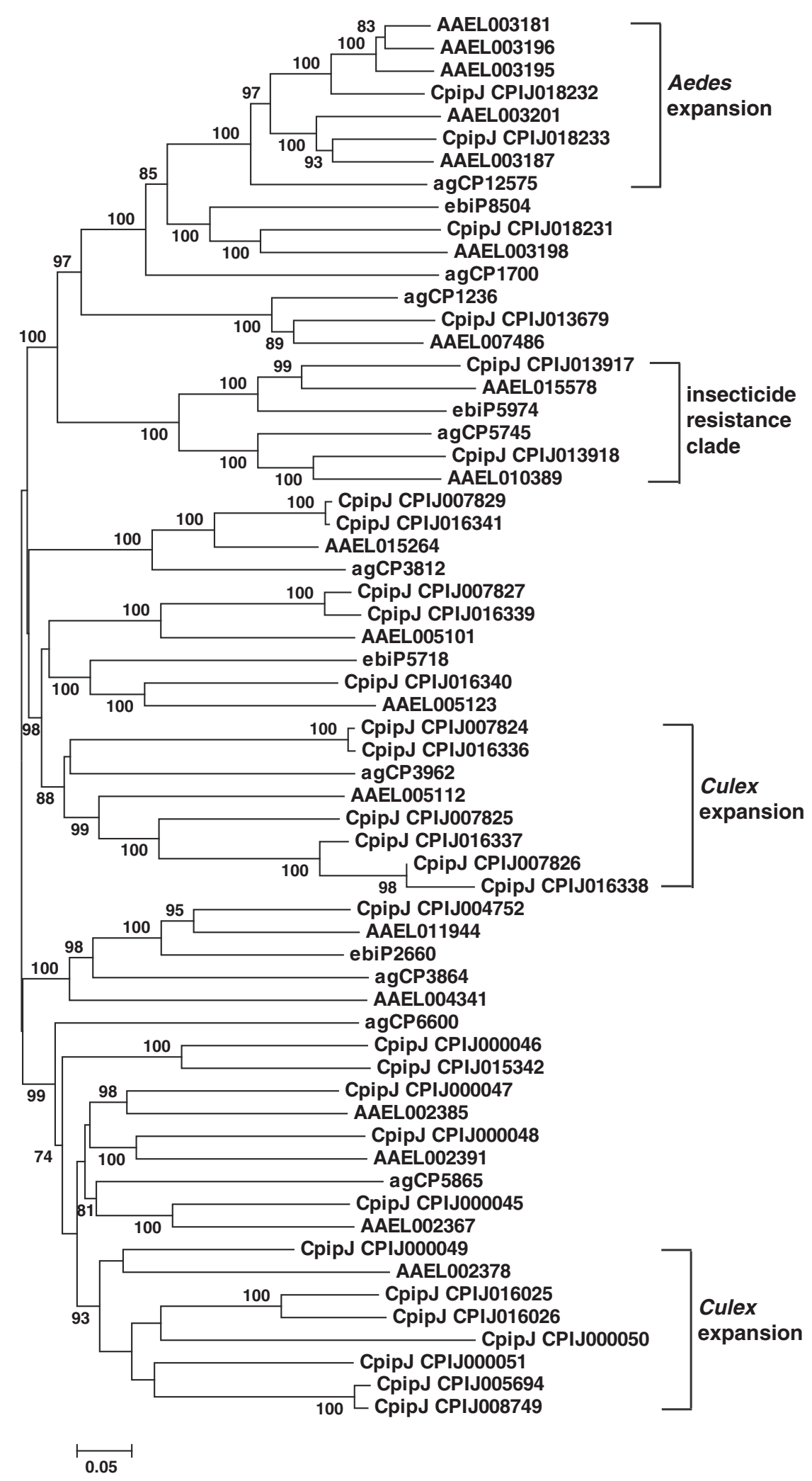

Figure 2 Unrooted distance neighbor-joining tree showing the phylogeny of a-esterases from the genome of Culex pipiens quinquefasciatus in relation to those from Aedes aegypti (initiated with 'AAEL') and Anopheles gambiae (initiated with 'agCP' or 'ebiP'). The percentage of bootstrap confidence values greater than 70\% (1000 replicates) is shown at the nodes. 
mosquito species, the expansion in the Culex happened in three clusters, which included five, three, and four JHEs, respectively, and two JHEs were specific for the Culex (Figure 3). Among 22 JHEs, 10 have typical GQSAG nucleophilic elbow motifs around the catalytic site and 6 have varied motifs, such as GH (W/N/Y) SAG. Empirical functional data are required to determine the identity of juvenile hormone esterases.

The secreted $\beta$-esterases (clade E) are comparatively conserved among the three mosquito species: from 2 to 4 - $\beta$-esterase genes were found in mosquito genomes, largely different from the expansion (11 $\beta$-esterase genes) found in N. vitripennis genome [9]. Some members of the $\beta$-esterases have well-described functions in other insects, such as E4 and FE4 esterases, which confer OP insecticide resistance in Myzus persicae [13], and the antennal Apo1PDE esterase of the silkworm Antheraea polyphemus, which degrades sex pheromones [14]. The functions of the $\beta$-esterases in Culex need to be further investigated.

Compared with the P450 genes of D. melanogaster and An. gambiae, those of C. p. quinquefasciatus expanded by $130 \%$ and $85 \%$, respectively, and were slightly more than the number of those in Ae. aegypti (Table 1). Expansion was most pronounced in the CYP4 clade, where, among 83 CYP4 genes, 46 belonged to CYP325 (Figure 4). Some CYP325s were conserved among the three mosquito species, such as CYP325E, CYP325K, and CYP325G; some only expanded in the Culex and Aedes genomes, such as CYP325X, CYP325Y; even Aedes and Anopheles had their specific CYP325s (Figure 5). However, Culex is not evolved speciesspecific large CYP325 gene expansion. The physiological function of the CYP325 clade in insects remains unclear, except that a CYP325A3 gene was found to be overexpressed in a permethrin-resistant strain of An. gambiae [15]. But this gene does not have clear orthologs in Culex or Aedes (Figure 5), indicating that the resistance mechanism of CYP325A3 overexpression may be limited to Anopheles. Other large clades of CYP4 genes included CYP4H, CYP4D, CYP4J, and CYP4C (Figure 4), members of which are involved in DDT and pyrethroid insecticide resistance in mosquitoes [15,16]. The P450 genes were also expanded in Ae. aegypti compared to An. gambiae, but this expansion was most prominent in the CYP9 of CYP3 family: 37 CYP9 genes in Ae. aegypti contrasting to just 9 in An. gambiae [15].

While the total number of CYP3 members (including CYP6 and CYP9) in C. $p$. quinquefasciatus was similar to that in Ae. aegypti, C. p. quinquefasciatus had more CYP6 members and few CYP9 members than Ae. aegypti [17]. Sixty-three CYP6 genes of C. p. quinquefasciatus were mainly distributed in the CYP6BY, CYP6N, CYP6M, CYP6AG, and CYP6Z groups (Figure 6A), and 25 CYP9 genes were mainly distributed in the CYP9J and CYP9M groups (Figure 6B). Members of CYP6 have been implicated in resistance to a broad range of insecticides (e.g., OPs, pyrethroids, DDT, and neonicotinoids) in many insects [15,18-20], while less evidence implicates CYP9s in the detoxification of insecticides. In 11 mitochondrial P450s, 7 genes belonged to CYP12F (Figure 6C). Members of the CYP12 clade are involved in DDT resistance in An. gambiae [15] and D. melanogaster [21]. Several large clusters of P450 genes were found in the C. p. quinquefasciatus genome, such as a cluster of 13 CYP9 genes on supercontig 278 and a cluster of 12 CYP6 genes on supercontig 869 (Additional file 2).

C. $p$. quinquefasciatus had 35 cytosolic GST genes belonging to the Delta, Epsilon, Omega, Sigma, and Theta clades (Table 1). The majority of the GSTs were represented by the Delta and Epsilon clades, which are insect-specific clades and contain the majority of the GSTs associated with detoxification in insecticides [22]. Many members of the Delta and Epsilon clades expanded locally in the C. p. quinquefasciatus genome, such as 12 Delta GSTs in supercontig 36 and 10 Epsilon GSTs in supercontig 1224 (Additional file 3). Delta 1, 6, and 7, and Epsilon 2, 4, and 8 showed 1:1:1 orthologies across the three mosquito genomes while Delta 11 and Epsilon 3 clades expanded on the Culex genome (Figure 7). Furthermore, Culex had specific Delta and Epsilon clades, which did not have clear orthologs in other two mosquito species (Figure 7). Unlike hymenopteran insects, where the Sigma class of GSTs expands and is thought to play an important role in protection against oxidative stress [9], only one Sigma GST gene was located in the genomes of dipteran insects. The Omega, Theta, and Zeta classes of GSTs are ubiquitously distributed in nature, but no Zeta GST has been identified in C. p. quinquefasciatus.

Why does C. p. quinquefasciatus have such an abundance of detoxification genes compared to other insect species? Several biological characteristics of mosquitoes may provide clues. The aquatic breeding sites of larvae and pupae contain numerous microorganisms, phenolic products of plant degradation, and pesticides. Adults feed on plant nectars and mammalian blood, which contain some harmful substances, such as heme and plant toxins. As viral pathogen vectors, mosquitoes have to deal with the generation of toxic endogenous compounds and reactive oxygen species during the immune response. But these cannot account for the gene expansion in C. p. quinquefasciatus compared to Anopheles and Aedes species. Perhaps its more polluted larval habitat and more diverse geographic range have exerted a greater selective pressure on C. $p$. quinquefasciatus so as to produce a larger repertoire of detoxification enzymes. 


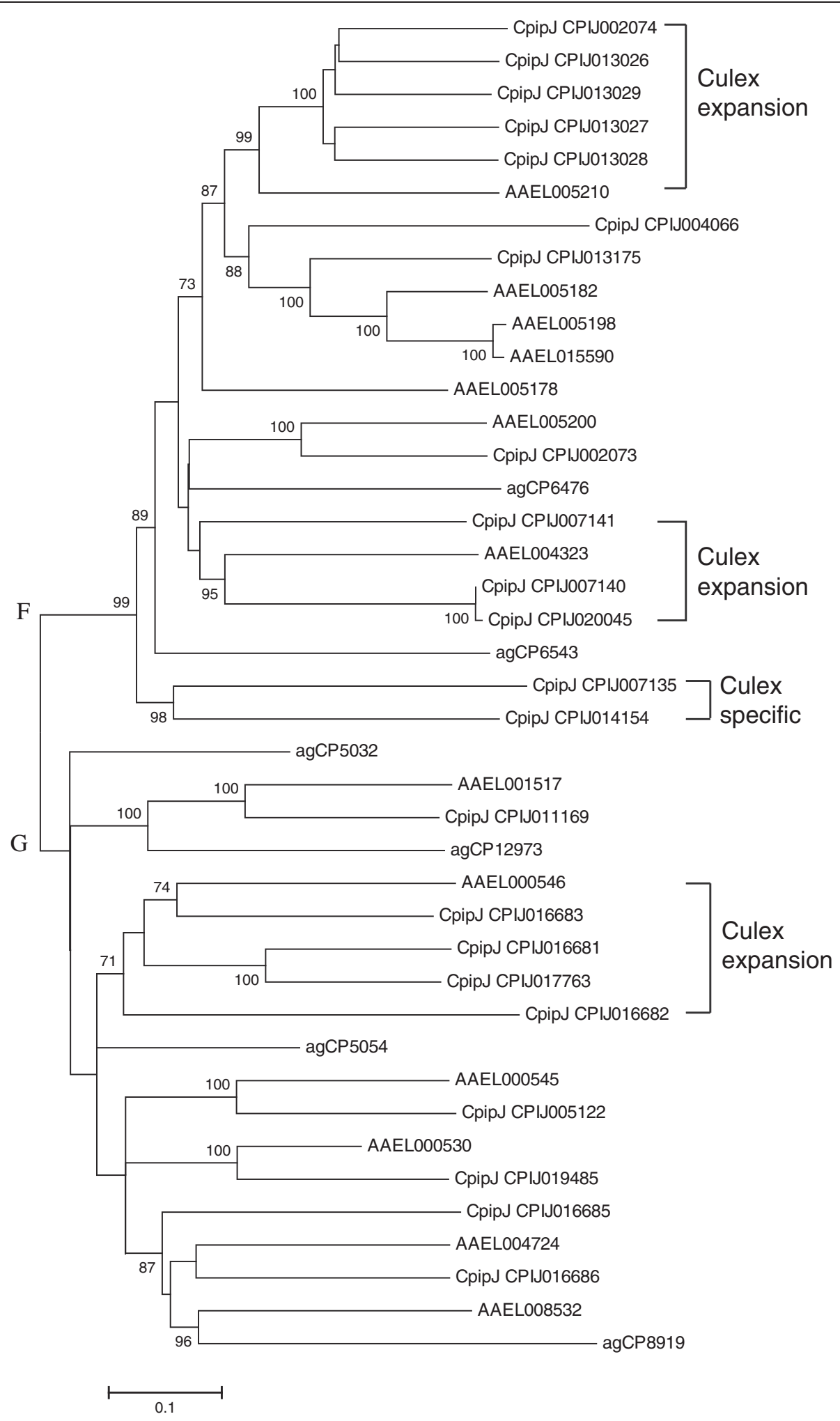

Figure 3 Unrooted distance neighbor-joining tree showing the phylogeny of juvenile hormone esterases from the genome of Culex pipiens quinquefasciatus in relation to those from Aedes aegypti (initiated with 'AAEL') and Anopheles gambiae (initiated with 'agCP'). The percentage of bootstrap confidence values greater than 70\% (1000 replicates) is shown at the nodes. 


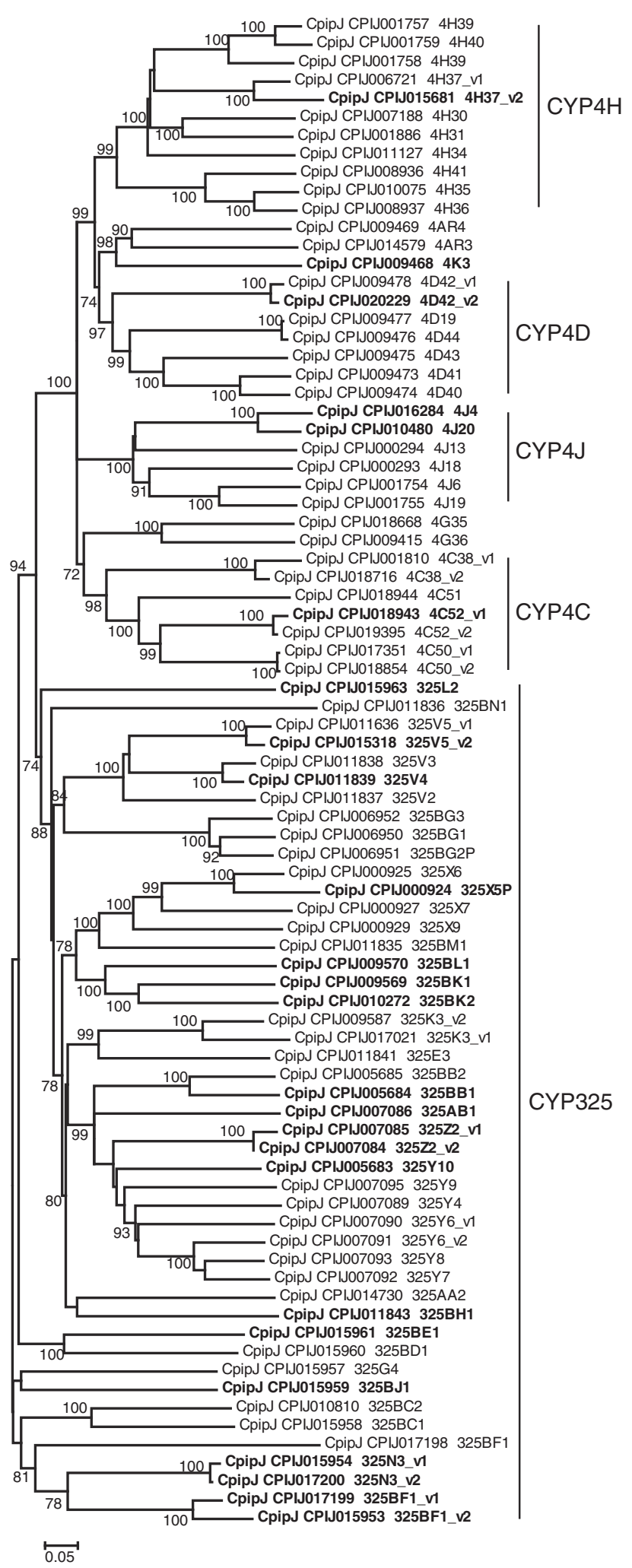

Figure 4 Unrooted distance neighbor-joining tree of P450 CYP4 genes from the genome of Culex pipiens quinquefasciatus. Greater than $70 \%$ support in 1000 bootstrap replications is indicated at the corresponding nodes. Genes undetected in DGE libraries are shown in bold. P450s were named by the P450 nomenclature committee (http://drnelson.uthsc.edu/CytochromeP450.html). Genes with v1 and v2 designation are very recent duplications and have not yet been assigned individual gene names. 
Figure 5 Unrooted distance neighbor-joining tree showing the phylogeny of P450 CYP325 genes from the genome of Culex pipiens quinquefasciatus (initiated with ' $\mathrm{CP}$ ') in relation to those from Aedes aegypti (initiated with 'AA') and Anopheles gambiae (initiated with 'AG'). The percentage of bootstrap confidence values greater than 70\% (1000 replicates) is shown at the nodes.

\section{DGE library sequencing and mapping to genome}

Using the DGE technique, which measures absolute, rather than relative, gene expression levels, the transcriptome was analyzed during the development of $C . p$. quinquefasciatus. For the five DGE libraries, 3.5 million to 3.8 million raw tags were generated (Table 2). The ratio of low quality reads was lower than $0.1 \%$ in all libraries except in that of the adult SG (Additional file 4). The number of distinct clean tags ranged from 113095 to 156922 (Table 2). The distribution of the total tags and distinct tags over different tag abundance showed similar patterns among the five libraries, indicating the normality of the DGE data (Figure 8). Highly expressed tags with copy numbers larger than 100 dominated in the distribution of the total clean tags, while tags with low expression and copy numbers smaller than 5 occupied the majority of the distinct clean tags (Figure 8). Pearson correlations between development stages ranged from 0.76 to 0.95 , indicating uneven transcriptome divergence during mosquito development or the existence of lowly expressed genes not detected, while the Pearson correlation between the two third instar larva libraries of the SG and S-lab strains was relatively high (0.98), reflecting the reproducibility of DGE sequencing (Additional file 5). Among 20306 reference genes in VectorBase, a total of 13314 (65.6\%) reference genes were mapped by unambiguous tags combining the five DGE libraries. Sequencing saturation analysis showed that the increase in the identified gene number nearly stopped when the number of reads reached 3 million (Additional file 6).

\section{GO and KEGG pathway classification of the genes}

\section{expressed in C. p. quinquefasciatus}

GO and KEGG pathway assignments were performed on the expressed genes to classify their functions and dissect the molecular events behind the expressed genes from the five DGE libraries. Of the 13314 genes, 2391 genes could be categorized into $48 \mathrm{GO}$ function groups, among which binding, catalytic activity, metabolic process, cellular process, and cell part or cell were predominant categories. In contrast, few genes were classified into groups for antioxidant activity, rhythmic process, pigmentation, cell wall organization, and carbon utilization (Figure 9). A total of 1629 genes were mapped to 125 KEGG pathways. The most-represented pathways 


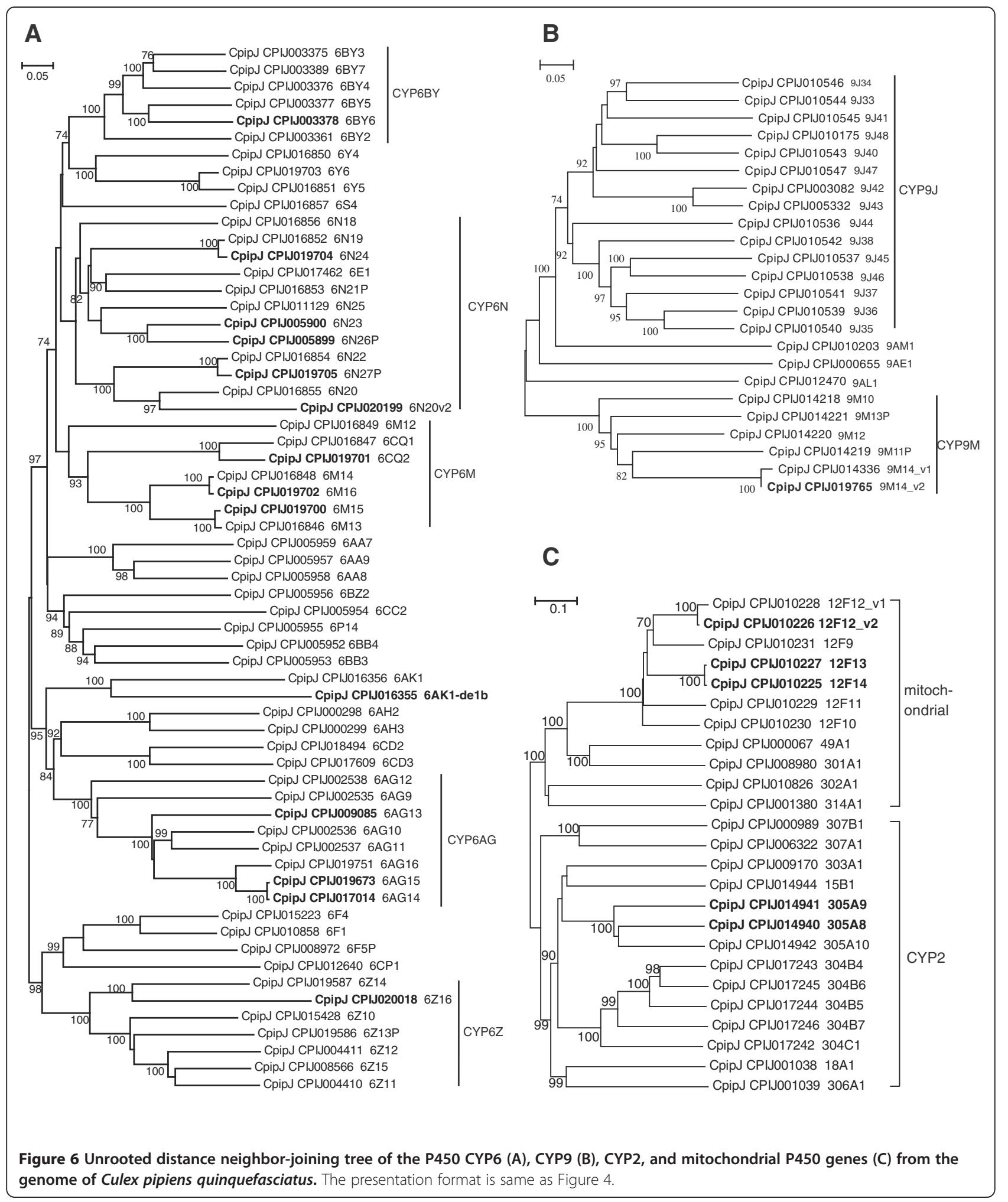

were metabolism (578 genes), microbial metabolism in diverse environments (101 genes), RNA transport (100 genes), spliceosome (95 genes), and protein processing in endoplasmic reticulum (95 genes).

\section{Life-stage specific detected genes}

Comparing the four DGE libraries of the SG strain, 2666 genes were detected in only one library, and their functions and involvement largely diverged (Additional file 7). 


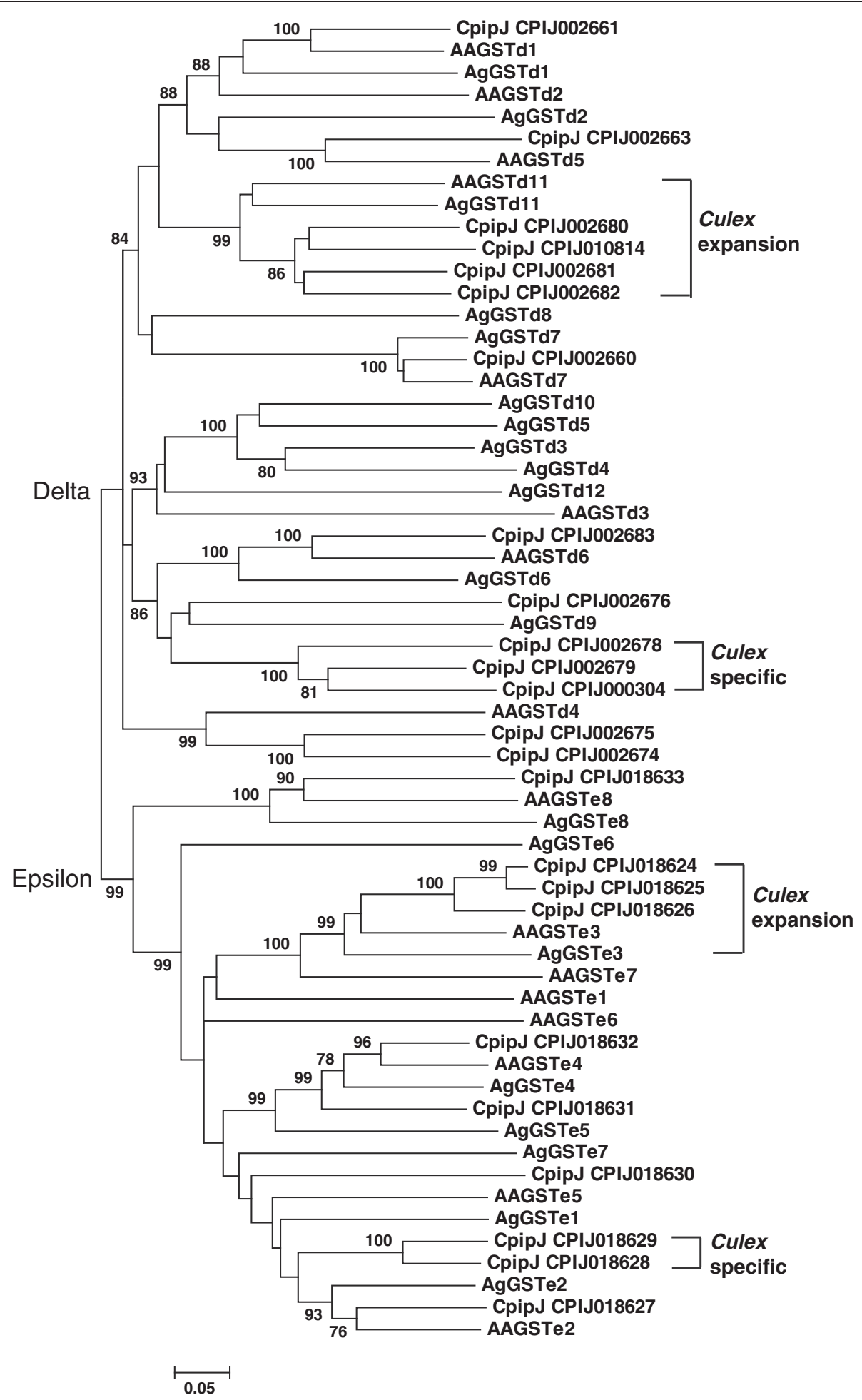

Figure 7 Unrooted distance neighbor-joining tree showing the phylogeny of delta and epsilon glutathione S-transferases from the genome of Culex pipiens quinquefasciatus in relation to those from Aedes aegypti (initiated with 'AA') and Anopheles gambiae (initiated with ' $\left.\mathbf{A} \mathbf{g}^{\prime}\right)$. The percentage of bootstrap confidence values greater than $70 \%$ (1000 replicates) is shown at the nodes. 
Table 2 DGE sequencing statistics

\begin{tabular}{|c|c|c|c|c|c|c|c|c|c|c|}
\hline & \multicolumn{8}{|c|}{ SG } & \multirow{2}{*}{\multicolumn{2}{|c|}{$\frac{\text { S-lab }}{\text { 3rd Larva }}$}} \\
\hline & \multicolumn{2}{|c|}{ Egg } & \multicolumn{2}{|c|}{ 3rd Larva } & \multicolumn{2}{|c|}{ Pupa } & \multicolumn{2}{|c|}{ Adult } & & \\
\hline & $\mathbf{N}$ & $\%$ & $\mathbf{N}$ & $\%$ & $\mathbf{N}$ & $\%$ & $\mathbf{N}$ & $\%$ & $\mathbf{N}$ & $\%$ \\
\hline Total raw tag & 3762501 & & 3790500 & & 3524500 & & 3769500 & & 3727501 & \\
\hline Total clean tag & 3759150 & & 3787346 & & 3521826 & & 3734683 & & 3724228 & \\
\hline Distinct clean tag & 156922 & & 118011 & & 132376 & & 113095 & & 143277 & \\
\hline Distinct tag mapping to gene & 59206 & 37.7 & 42706 & 36.2 & 45666 & 34.5 & 41254 & 36.5 & 51264 & 35.8 \\
\hline Distinct unambiguous tag mapping to gene & 48378 & 30.8 & 34772 & 29.5 & 37693 & 28.5 & 34449 & 30.5 & 39475 & 27.6 \\
\hline Unambiguous tag-mapped genes & 10917 & 53.8 & 9808 & 48.3 & 10252 & 50.5 & 9759 & 48.1 & 10976 & 54.1 \\
\hline Distinct tag mapping to genome & 48679 & 31.0 & 36533 & 31.0 & 40063 & 30.3 & 35062 & 31.0 & 47035 & 32.8 \\
\hline Total unknown tag & 424236 & 11.3 & 397001 & 10.5 & 442991 & 12.6 & 415816 & 11.1 & 355720 & 9.6 \\
\hline Distinct unknown tag & 49037 & 31.2 & 38772 & 32.9 & 46647 & 35.2 & 36779 & 32.5 & 44978 & 31.4 \\
\hline
\end{tabular}

For each number $(\mathrm{N})$ of tags or tag-mapped genes, the associated percentage (\%) regarding the distinct clean tags (156922), the reference genes (20306), or total clean tags (3759150) is indicated.

Distribution of total clean tags

Tag copy number

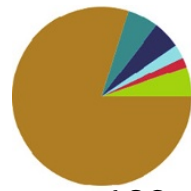

egg of SG

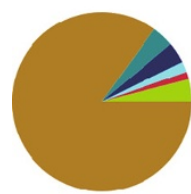

larva of $S G$

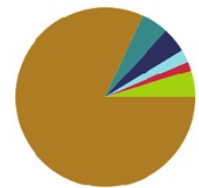

pupa of SG

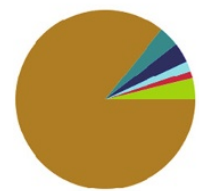

adult of $S G$

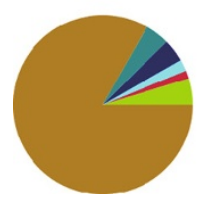

larva of S-lab > $>100(3099299,83.22 \%)$

[1, 5] (198910, 5.29\%)

- $[6,10](68659,1.83 \%)$

- $[11,20](93150,2.48 \%)$

- $[21,50](185820,4.94 \%)$

[51, 100] $(211529,5.63 \%)$

$>100(3001082,79.83 \%)$

Tag copy number

$[1,5](153229,4.05 \%)$

$[6,10](51255,1.35 \%)$

$[11,20](72653,1.92 \%)$

- $[21,50](142311,3.76 \%)$

[ $[51,100](149173,3.94 \%)$

$>100(3218725,84.99 \%)$

Tag copy number

$[1,5](167403,4.75 \%)$

ㅁ $[6,10](59042,1.68 \%)$

- $[11,20](80450,2.28 \%)$

- $[21,50](156769,4.45 \%)$

$[51,100](174149,4.94 \%)$

$>100$ (2884013, 81.89\%)

Tag copy number

$[1,5](142098,3.80 \%)$

- $[6,10](49919,1.34 \%)$

$[11,20](68333,1.83 \%)$

[ $[21,50](132511,3.55 \%)$

$[51,100](137572,3.68 \%)$

$>100(3204250,85.80 \%)$

Tag copy number

$[1,5](177233,4.76 \%)$

- $[6,10](54131,1.45 \%)$

[11, 20] $(77246,2.07 \%)$

$[21,50](153349,4.12 \%)$

$[51,100](162970,4.38 \%)$
Distribution of distinct clean tags

Tag copy number
$[1,5](128558,81.92 \%)$

口 $[6,10](9056,5.77 \%)$

$[11,20](6332,4.04 \%)$

- $[21,50](5761,3.67 \%)$

$[51,100](2972,1.89 \%)$

$>100(4243,2.70 \%)$

egg of SG

Tag copy number

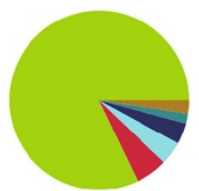

$[1,5](96918,82.13 \%)$

ㅁ $[6,10](6763,5.73 \%)$

$[11,20](4965,4.21 \%)$

[21, 50] (4439, 3.76\%)

$[51,100](2108,1.79 \%)$

larva of $S G \square>100(2818,2.39 \%)$

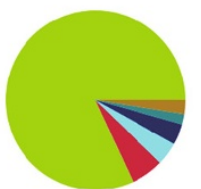

Tag copy number

$[1,5](108472,81.94 \%)$

[ $[6,10](7775,5.87 \%)$

$[11,20](5445,4.11 \%)$

$[21,50](4884,3.69 \%)$

$[51,100](2460,1.86 \%)$

pupa of SG > $\quad 100(3340,2.52 \%)$

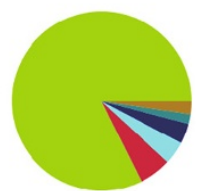

Tag copy number

$[1,5](93134,82.35 \%)$

口 $[6,10](6553,5.79 \%)$

ㅁ $[11,20](4691,4.15 \%)$

- $[21,50](4113,3.64 \%)$

$[51,100](1965,1.74 \%)$

adult of SG $\quad>100(2639,2.33 \%)$

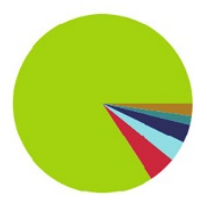

Tag copy number

$[1,5](120638,84.20 \%)$

$[6,10](7117,4.97 \%)$

$[11,20](5264,3.67 \%)$

$[21,50](4807,3.36 \%)$

$[51,100](2292,1.60 \%)$

larva of S-lab > $100(3159,2.20 \%)$

Figure 8 Distribution of total clean tags and distinct clean tags over different tag abundance in each DGE library. Numbers in square brackets indicate the range of copy numbers for a specific category of tags. Numbers in parentheses show the total number of tags in that category. 
Among 302 detoxification genes, 225 (75\%) were detected for expression in at least one DGE library (Table 1). About $25 \%$ of the genes that were not detected in any library were either not constitutively expressed in the life stages or were possibly untranscribed pseudogenes. Among expressed detoxification genes, $30 \%$ of the CCE genes, $25 \%$ of the P450 genes, and $72 \%$ of the GST genes were expressed in all life stages, indicating that they play a general housekeeping or detoxification function; some detoxification genes were developmentalstage specific (Table 3). Around $28 \%$ of the CCE genes were detected in only one stage, and two $\alpha$-esterases, CpipJ CPIJ018232 and CpipJ CPIJ004752, were expressed at extremely high levels in larvae and pupae, respectively. Two insecticide-resistance-responsible esterases, CpipI_ CPIJ013917 for encoding esterase B and CpipJ CPIJ013918 for encoding esterase A, were expressed in all life stages, and their expression levels in larvae were higher than in adults. Only two GST genes showed stage-specific expression. Of the P450 genes, around $25 \%$ were detected in only one stage and most of them belonged to CYP4. Most strikingly, of 46 CYP325 genes, representing the widest expansion within the P450 family, 27 genes (59\%) were detectable for expression and nearly half of the expressed genes (12 CYP325) were developmentally regulated. The larva and adult are feeding stages while the egg and pupae are non-feeding. Hence enzymes expressed specifically or highly in larva or adult are important in digestion and detoxification of dietary component whereas those in egg or pupae are vital for detoxification of metamorphosis byproducts or synthesis of specific hormones.

For gene expansion clusters of detoxification genes, the expression profiles were different among the members. For example, among the six members of one expanded $\alpha$-esterase cluster (Figure 2), CpipJ_CPIJ007825 was detected for expression in pupae and adults while the other five members were not detected in any stage. For the seven members of another expanded $\alpha$-esterase cluster (Figure 2), CpipJ_CPIJ016025, CpipJ_CPIJ005694, and CpipJ_CPIJ008749 were not detected for expression;

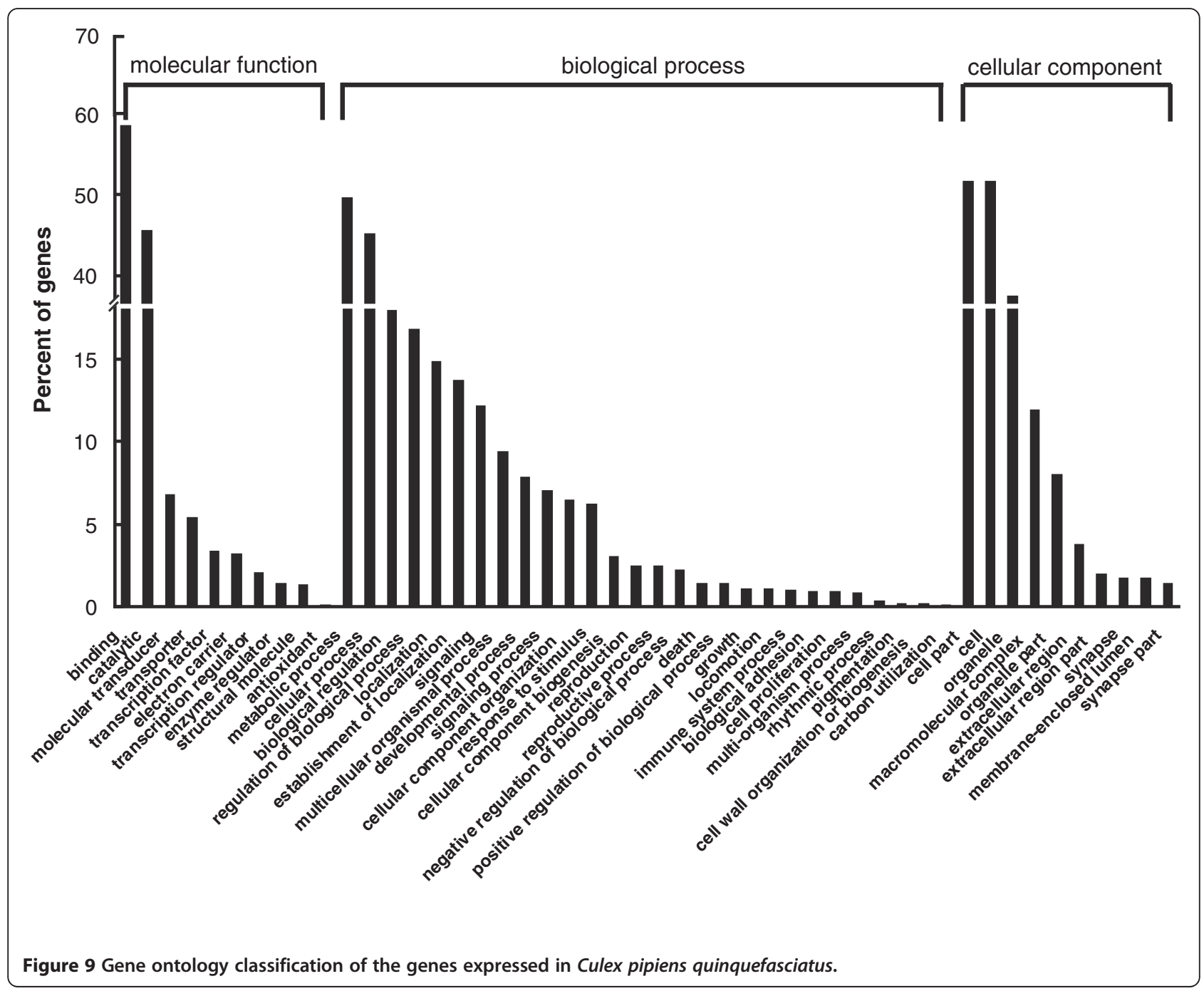


Table 3 Developmental-stage specifically expressed genes of carboxylcholinesterase (CCE), glutathione-Stransferase (GST), and cytochrome P450 monooxygenase (P450)

\begin{tabular}{|c|c|c|c|c|}
\hline Family & Stage & Gene & Class & TPM \\
\hline \multirow[t]{13}{*}{ CCE } & Egg & CpipJ_CPIJ007135 & $\mathrm{F}$ & 0.27 \\
\hline & & CpipJ_CPIJ016026 & B & 0.27 \\
\hline & & CpipJ_CPIJ009768 & M & 0.53 \\
\hline & & CpipJ_CPIJ013027 & $\mathrm{F}$ & 0.53 \\
\hline & Larva & CpipJ_CPIJ016341 & B & 0.26 \\
\hline & & CpipJ_CPIJ016682 & G & 2.00 \\
\hline & & CpipJ_CPIJ018231 & B & 5.81 \\
\hline & Pupa & CpipJ_CPIJ018232 & B & 32.74 \\
\hline & & CpipJ_CPIJ013679 & $B$ & 0.57 \\
\hline & & CpipJ_CPIJ013175 & $\mathrm{F}$ & 13.63 \\
\hline & Adult & CpipJ_CPIJ004752 & B & 118.69 \\
\hline & & CpipJ_CPIJ016681 & G & 0.54 \\
\hline & & CpipJ_CPIJ005122 & G & 5.62 \\
\hline \multirow[t]{2}{*}{ GST } & Larva & CpipJ_CPIJ002674 & Delta & 3.96 \\
\hline & & CpipJ_CPIJ014051 & Theta & 7.66 \\
\hline \multirow[t]{29}{*}{ P450 } & Egg & CpipJ_CPIJ019395 & CYP4C52 v2 & 0.27 \\
\hline & & CpipJ_CPIJ009478 & CYP4D42 v1 & 0.27 \\
\hline & & CpipJ_CPIJ017198 & CYP325BF1 & 0.27 \\
\hline & & CpipJ_CPIJ003361 & CYP6BY2 & 0.80 \\
\hline & & CpipJ_CPIJ017244 & CYP304B5 & 2.00 \\
\hline & Larva & CpipJ_CPIJ009474 & CYP4D40 & 0.26 \\
\hline & & CpipJ_CPIJ010228 & CYP12F12 v1 & 0.26 \\
\hline & & CpipJ_CPIJ015428 & CYP6Z10 & 0.26 \\
\hline & & CpipJ_CPIJ001755 & CYP4J19 & 0.26 \\
\hline & & CpipJ_CPIJ014220 & CYP9M12 & 0.53 \\
\hline & & CpipJ_CPIJ014219 & CYP9M11P & 1.06 \\
\hline & & CpipJ_CPIJ014730 & CYP325AA2 & 1.06 \\
\hline & & CpipJ_CPIJ007188 & CYP4H30 & 1.32 \\
\hline & & CpipJ_CPIJ007091 & CYP325Y6 v2 & 1.58 \\
\hline & & CpipJ_CPIJ011127 & CYP4H34 & 1.85 \\
\hline & & CpipJ_CPIJ010075 & CYP4H35 & 2.11 \\
\hline & & CpipJ_CPIJ003376 & CYP6BY4 & 2.64 \\
\hline & & CpipJ_CPIJ007089 & CYP325Y4 & 4.22 \\
\hline & & CpipJ_CPIJ015223 & CYP6F4 & 6.6 \\
\hline & & CpipJ_CPIJ010858 & CYP6F1 & 47.0 \\
\hline & & CpipJ_CPIJ014942 & CYP305A10 & 1.00 \\
\hline & Pupa & CpipJ_CPIJ001754 & CYP4J6 & 0.28 \\
\hline & & CpipJ_CPIJ007095 & CYP325Y9 & 0.28 \\
\hline & & CpipJ_CPIJ005685 & CYP325BB2 & 0.28 \\
\hline & & CpipJ_CPIJ010810 & CYP325BC2 & 0.28 \\
\hline & & CpipJ_CPIJ004410 & CYP6Z11 & 0.85 \\
\hline & & CpipJ_CPIJ009477 & CYP4D19 & 1.42 \\
\hline & Adult & CpipJ_CPIJ019587 & CYP6Z14 & 0.27 \\
\hline & & CpipJ_CPIJ006951 & CYP325BG2P & 0.27 \\
\hline
\end{tabular}

Table 3 Developmental-stage specifically expressed genes of carboxylcholinesterase (CCE), glutathione-Stransferase (GST), and cytochrome P450 monooxygenase (P450) (Continued)

\begin{tabular}{lllr}
\hline CpipJ_CPIJ010542 & CYP9J38 & 0.27 \\
\cline { 2 - 4 } & CpipJ_CPIJ003377 & CYP6BY5 & 0.54 \\
\cline { 2 - 4 } CpipJ_CPIJ015960 & CYP325BD1 & 1.87 \\
\cline { 2 - 4 } CpipJ_CPIJ011837 & CYP325V2 & 2.68 \\
\cline { 2 - 4 } & CpipJ_CPIJ019586 & CYP6Z13P & 2.68 \\
\cline { 2 - 4 } & CpipJ_CPIJ006950 & CYP325BG1 & 3.21 \\
\cline { 2 - 4 } CpipJ_CPIJ010203 & CYP9AM1 & 5.36 \\
\cline { 2 - 4 } & CpipJ_CPIJ015957 & CYP325G4 & 51.68 \\
\cline { 2 - 4 } & CpipJ_CPIJ009471 & CYP4AR4 & 2.00 \\
\hline
\end{tabular}

TPM, number of transcripts per million clean tags.

CpipJ_CPIJ000049 and CpipJ_CPIJ000051 were expressed in pupae and adults, CpipJ_CPIJ000050 in larvae and pupae, and CpipJ_CPIJ016026 only in eggs. Similar phenomenon was observed in the three expansion clusters of juvenile hormone esterases (Figure 3). For the five members of cluster, only CpipJ_CPIJ013027 was detected for expression and in eggs. No expression was found in the three members of cluster. For the four members of cluster, three were detected for expression: CpipJ_ CPIJ016681 and CPipJ_CPIJ016682 in adults, and CpipJ_CPIJ017763 in larvae and adults. The different expression patterns of these duplicated detoxification genes are probably indicative of their subfunctionalization or retrogression as pseudogenes.

\section{Differentially expressed genes between parathion resistant and susceptible larvae}

When the third instar DGE library of the parathionresistant strain SG was compared with the same stage in the susceptible strain S-lab, a total of 1511 genes showed different expression levels, among which 619 genes had up-regulated expression levels in the SG strain (Additional file 8). The most prominent GO functions of these up-regulated genes were endopeptidase or serinetype peptidase activity, such as genes encoding trypsin, chymotrypsin, mast cell protease 2, urokinase-type plasminogen activator, and elastase. However, not all of the differentially expressed genes are responsible for parathion resistance because comparison strains were not selected from the same panmictic population such that genetic background differences could be ruled out.

A total of 15 detoxification genes were expressed at higher levels in the SG strain, including 2 CCEs, 6 GSTs, and 7 P450s (Table 4). The expression of the known esterase B gene, CpipJ CPIJ013917, increased 16-fold in the resistant strain, while the esterase A gene, CpipJ CPIJ013918, did not show differential expression between the two strains. Three Epsilon GSTs had 
prominent involvement in resistance, as previously reported in DDT- and OP-resistant mosquitoes and houseflies [22,23]. Seven P450 genes with elevated expression in the resistant strain belonged to either the CYP9 or CYP6 groups, which are usually implicated in pyrethroid resistance in mosquitoes and other species [18]. P450 monooxygenases are relatively less commonly involved than carboxylesterases in resistance to OP insecticides, although evidence shows that CYP6A1 from Musca domestica and CYP6A2 from Drosophila are capable of metabolizing diazinon [24,25]. Unless direct evidence demonstrates the detoxification or sequestration of insecticide compounds by $\mathrm{P} 450 \mathrm{~s}$, the conclusion that the 7 up-regulated $\mathrm{P} 450$ genes are involved in OP resistance is too early to draw. Most of the 15 detoxification genes were expressed in all life stages, while 6 were expressed only in certain stages, especially Theta GST (CpipJ CPIJ014051), CYP9J34 (CpipJ CPIJ010546), and CYP6F1 (CpipJ CPIJ010858), which were not found in adults. The different responses of detoxification genes may account for the different resistance levels between the larval and adult stage in some circumstances.

\section{Conclusion}

C. p. quinquefasciatus is an important vector that transmits human diseases different from those by An. gambiae and Ae. aegypti. The lack of transcriptomic data available for this species has hampered characterization of the molecular mechanisms underlying the different biological characters of the three major mosquito vectors. The five DGE libraries described in the present study represent a dramatic expansion of the existing transcriptomic sequence available for C. p. quinquefasciatus. This expansion will facilitate the investigation of the fundamental biology of $C$. p. quinquefasciatus and its pathogenic interactions. In addition, the results of the present study provide new insights into the functions and evolution of the three detoxification gene families of mosquitoes. A larger number of detoxification genes were identified on the genome of $C$. p. quinquefasciatus compared with three other dipteran insect genomes, representing the widest gene expansion sequenced thus far. Comparative genomic analysis suggested that gene expansion mainly occurs in $\alpha$-esterases, juvenile hormone esterases, and P450 CYP325. Some detoxification genes were expressed in all developmental stages, while some were developmentally regulated. The expression profiles were different among the members of gene expansion clusters, probably indicative of their subfunctionalization or retrogression as pseudogenes. Fifteen detoxification genes showed the potential to take part in the parathion resistance of Culex, including unexpected P450 genes.

\section{Methods \\ Mosquito strains}

Mosquito strains of C. p. quinquefasciatus used included $\mathrm{S}$-lab, which was OP-susceptible and reared at the laboratory without any contact with insecticides for many years [26] and Shengui (SG), a field population collected in Foshan, Guangdong Province, in 2007 and constantly treated with parathion at the laboratory. The parathionresistance of SG was 115-fold that of S-lab before use in the DGE analysis. The mosquitoes were maintained at $26^{\circ} \mathrm{C} \pm 1{ }^{\circ} \mathrm{C}$ and a long-day photoperiod (14 h light/10 $\mathrm{h}$ darkness cycle). Fifty egg rafts, forty third instar larvae, forty pupae, and forty adults (twenty females and twenty

Table 4 Detoxification genes up-regulated in parathion resistant larvae of the SG strain

\begin{tabular}{|c|c|c|c|c|}
\hline Gene family & Classification & Gene number & $\log _{2}$ Ratio $^{*}$ & Expression stage \\
\hline \multirow[t]{2}{*}{ CCE } & a esterases & CpipJ_CPIJ013917 & 4.4 & All \\
\hline & $\mathrm{JH}$ esterases & CpipJ_CPIJ002073 & 1.7 & All \\
\hline \multirow[t]{6}{*}{ GST } & Epsilon & CpipJ_CPIJ018629 & 8.7 & All \\
\hline & Epsilon & CpipJ_CPIJ018632 & 5.0 & All \\
\hline & Epsilon & CpipJ_CPIJ018627 & 3.5 & All \\
\hline & Theta & CpipJ_CPIJ014051 & 2.2 & Larva \\
\hline & Delta & CpipJ_CPIJ002675 & 1.9 & Egg, larva, adult \\
\hline & Others & CpipJ_CPIJ014694 & 1.1 & All \\
\hline \multirow[t]{7}{*}{ P450 } & CYP9J34 & CpipJ_CPIJ010546 & 4.0 & Larva, pupa \\
\hline & CYP9J40 & CpipJ_CPIJ010543 & 3.3 & Larva, pupa, adult \\
\hline & CYP6AG11 & CpipJ_CPIJ002537 & 3.0 & All \\
\hline & CYP6BZ2 & CpipJ_CPIJ005956 & 2.6 & All \\
\hline & CYP9AE1 & CpipJ_CPIJ000655 & 2.0 & All \\
\hline & CYP6F1 & CpipJ_CPIJ010858 & 1.3 & Larva \\
\hline & CYP9AL1 & CpipJ_CPIJ012470 & 1.1 & Larva, adult \\
\hline
\end{tabular}

*Ratio, TPM of SG/TPM of Slab. TPM, number of transcripts per million clean tags. 
males) of SG and forty third instar larvae of S-lab were collected and frozen at $-80^{\circ} \mathrm{C}$ for further analysis.

\section{Identification and phylogenetic classification of detoxification genes}

Sequences encoding GSTs, P450s, and CCEs were identified from the protein set of the C. $p$. quinquefasciatus whole genome sequencing database at the Broad Institute (http://www.broadinstitute.org/annotation/genome/ culex_pipiens) using the HMMER program (http://hmmer. janelia.org/) with the protein domains for CCEs (PF00135), GSTs (PF00043 and PF02798), and P450s (PF00067) as described in the Pfam database. A significance value of $1 \mathrm{e}^{-10}$ was used in the searches for CCEs and P450 and $2 \mathrm{e}^{-2}$ for GSTs. Community annotations and VectorBase were referred to verify the searches [27]. Those candidate genes not supported by the community annotations as CCEs, P450 or GST were not accounted. P450s were named by the P450 nomenclature committee (http:// drnelson.uthsc.edu/CytochromeP450.html). Known detoxification genes from An. gambiae, D. melanogaster [28], and Ae aegypti [17] were used as references for the phylogenetic classification of the detoxification genes from C. $p$. quinquefasciatus. Protein sequences were aligned with ClustalW2 at EMBL-EBI (http://www.ebi. ac.uk/Tools/msa/clustalw2). Unrooted distance neighborjoining trees showing the phylogeny of detoxification gene families were constructed using the pairwise deletion and p-distance functions of Mega 4.0 software. Bootstrap analysis (1000 replicates) was applied to evaluate the internal support of the tree topology.

\section{Pipeline of DGE}

Six micrograms of total RNA from each of the above five mosquito samples were isolated using TRIzol reagent (Invitrogen, Carlsbad, CA, USA) according to the manufacturer's instructions. Tag library preparation was performed with an Illumina Gene Expression Sample Prep Kit. The raw data (tag sequences and counts) were deposited in the NCBI Sequence Read Archive (SRA) database under submission number SRA049959.

\section{Pipeline of bioinformatics analysis on DGE}

Sequencing-received raw image data were transformed by base calling into raw sequence data. Clean tags were obtained after raw sequences were filtered to remove adaptor sequences, empty tags, low quality tags, tags that were too long or too short, and tags with a copy number of 1 . The distribution of clean tags was used to evaluate the normality of the whole data. Saturation analysis was performed to determine whether or not the number of detected genes continues to increase when the sequencing amount increases. Pearson correlation analysis of two parallel libraries was performed to evaluate the reliability and operational stability of the experimental results. All clean tags were mapped to C. p. quinquefasciatus whole genome reference sequences and allowed no more than 1 nucleotide mismatch. The number of unambiguous clean tags for each gene was calculated and then normalized to TPM (number of transcripts per million clean tags). When the expression of a gene was not detected, TPM was set to 0.01 .

A rigorous custom written algorithm using the method described by Audic et al. [29] was developed to identify differentially expressed genes between two samples. The $\mathrm{p}$ value corresponded to the differential gene expression test. False discovery rate (FDR) was used to determine the $\mathrm{p}$ value threshold in multiple tests and analyses [30]. FDR $\leq 0.001$ and the absolute value of $\log _{2}$ Ratio $\geq 1$ were used as thresholds to judge the significance of the gene expression difference.

Unigenes matched by clean tags were assigned to Gene Ontology (GO) terms using Blast2GO and canonical pathways in KEGG (Kyoto Encyclopedia of Genes and Genomes). GO or pathway enrichment analysis of the differentially expressed genes was performed based on the algorithm presented by GOstat [31]. The difference between the differentially expressed gene group and the whole gene expression background was represented by a $\mathrm{p}$ value, which was approximated by a chi-square test. The Fisher exact test was used when any expected count value was below 5 , which will result in inaccurate chisquare test results. Benjamini multiple-testing correction of the $\mathrm{p}$ value was done by FDR.

\section{Additional files}

Additional file 1: Summary of the carboxylcholinesterase genes of Culex pipiens quinquefasciatus. TPM, number of transcripts per million clean tags. When the expression of a gene was not detected, TPM was set to 0.01 .

Additional file 2: Summary of the cytochrome P450 monooxygenase genes of Culex pipiens quinquefasciatus. TPM, see Additional file 1.

Additional file 3: Summary of the glutathione S-transferase genes of Culex pipiens quinquefasciatus. TPM, see Additional file 1.

Additional file 4: Distribution of the total tags in each DGE library. Additional file 5: Pearson correlation analysis of the DGE libraries. Dots in the figures indicate individual tag entities. TPM (Transcripts Per Million clean tags) indicates the number of transcript copies in every 1 million clean tags. A-F, correlation between the four developmental stages of the SG strain; G, correlation between third instar larvae of the SG and S-lab strains.

Additional file 6: Relationship between the number of identified genes and sequencing amount.

Additional file 7: Enriched GO function groups ${ }^{*}$ and KEGG pathways involved by genes specifically expressing in various life stages of Culex pipiens quinquefasciatus. " $\mathrm{GO}$ function groups include three main categories: biological process (BP), molecular function (MF), and cellular component (CC). ${ }^{\dagger}$ The $p$ value represented the difference between the specifically expressed gene group and the total 13,314 gene group approximated by chi-square test. Fisher exact test is used when 
any expected count value is below $5 .{ }^{~}$ Benjamini is the multiple-testing correction of the $p$ value by FDR.

Additional file 8: Differentially expressed genes between the parathion-resistant and susceptible larvae of Culex pipiens quinquefasciatus. TPM, see Additional file 1.

\section{Competing interests}

The authors declare that they have no competing interests.

\section{Authors' contributions}

LY and NC prepared the RNA, performed the phylogenetic analysis of the detoxification genes, and drafted the manuscript. PY and FJ performed the GO and pathway enrichment analysis of differentially expressed genes. EM and CQ conceived and designed the experiment. FC designed the experiment, performed the phylogenetic analysis of the detoxification genes, and wrote the manuscript. All authors read and approved the final manuscript.

\section{Acknowledgements}

This study was financially supported by the Major State Basic Research Development Program of China (973 Program, Grant No. 2012CB114102), the Important National Science \& Technology Specific Project (Grant No. 2012ZX10004219), and the Knowledge Innovation Program of Chinese Academy of Sciences (Grant No. KSCX3-IOZ-1006).

\section{Author details}

${ }^{1}$ State Key Laboratory of Integrated Management of Pest Insects \& Rodents, Institute of Zoology, Chinese Academy of Sciences, Beijing 100101, China. ${ }^{2}$ Research Institute of Applied Biology, Shanxi University, Taiyuan, Shanxi 030006, China.

Received: 29 June 2012 Accepted: 8 November 2012 Published: 10 November 2012

\section{References}

1. Liu N, Xu Q, Zhu F, Zhang L: Pyrethroid resistance in mosquitoes. Insect Sci 2006, 13:159-166.

2. Grant DF, Dietze EC, Hammock BD: Glutathione Stransferase isozymes in Aedes aegypti: purification, characterization, and isozyme-specific regulation. Insect Biochem 1991, 21:421-433.

3. Raymond M, Chevillon C, Guillemaud T, Lenormand T, Pasteur N: An overview of the evolution of overproduced esterase in mosquito Culex pipiens. Philos Trans R Soc Lond B 1998, 353:1701-1711.

4. Arensburger P, Megy K, Waterhouse RM, Abrudan J, Amedeo P, Antelo B, Bartholomay L, Bidwell S, Caler E, Camara F, Campbell CL, Campbell KS, Casola C, Castro MT, Chandramouliswaran I, Chapman SB, Christley S, Costas J, Eisenstadt E, Feschotte C, Fraser-Liggett C, Guigo R, Haas B, Hammond M, Hansson BS, Hemingway J, Hill SR, Howarth C, Ignell R, Kennedy RC, et al: Sequencing of Culex quinquefasciatus establishes a platform for mosquito comparative genomics. Science 2010, 330:86-88.

5. Holt RA, Subramanian GM, Halpern A, Sutton GG, Charlab R, Nusskern DR, Wincker P, Clark AG, Ribeiro JMC, Wides R, Salzberg SL, Loftus B, Yandell M, Majoros WH, Rusch DB, Lai Z, Kraft CL, Abril JF, Anthouard V, Arensburger P, Atkinson PW, Baden H, de Berardinis V, Baldwin D, Benes V, Biedler J, Blass C, Bolanos R, Boscus D, Barnstead M, et al: The genome sequence of the malaria mosquito Anopheles gambiae. Science 2002, 298:129-149.

6. Nene V, Wortman JR, Lawson D, Haas B, Kodira C, Tu Z, Loftus B, Xi Z, Megy K, Grabherr M, Ren Q, Zdobnov EM, Lobo NF, Campbell KS, Brown SE, Bonaldo MF, Zhu J, Sinkins SP, Hogenkamp DG, Amedeo P, Arensburger P, Atkinson PW, Bidwell S, Biedler J, Birney E, Bruggner RV, Costas J, Coy MR, Crabtree J, Crawford M, et al: Genome sequence of Aedes aegypti, a major arbovirus vector. Science 2007, 316:1718-1723.

7. Girard YA, Mayhew GF, Fuchs JF, Li H, Schneider BS, McGee CE, Rocheleau TA, Helmy H, Christensen BM, Higgs S, Bartholomay LC: Transcriptome changes in Culex quinquefasciatus (Diptera: Culicidae) salivary glands during West Nile virus infection. J Med Entomol 2010, 47:421-435.

8. Ribeiro JM, Charlab R, Pham VM, Garfield M, Valenzuela JG: An insight into the salivary transcriptome and proteome of the adult female mosquito Culex pipiens quinquefasciatus. Insect Biochem Mol Biol 2004, 34:543-563.
9. Oakeshott JG, Johnson RM, Berenbaum MR, Ranson H, Cristino AS, Claudianos C: Metabolic enzymes associated with xenobiotic and chemosensory responses in Nasonia vitripennis. Insect Mol Biol 2010, 19(Suppl. 1):147-163.

10. Newcomb RD, Campbell PM, Ollis DL, Cheah E, Russell RJ, Oakeshott JG: A single amino acid substitution converts a carboxylesterase to an organophosphorus hydrolase and confers insecticide resistance on a blowfly. Proc Natl Acad Sci USA 1997, 94:7464-7468.

11. Korochkin LI, Ludwig MZ, Poliakova EV, Philinova MR: Some molecular genetic aspects of cellular differentiation in Drosophila. Soviet Sci Rev 1987, 1:411-466.

12. Pasteur N, Iseki A, Georghiou GP: Genetic and biochemical studies of the highly active esterases $A^{\prime}$ and $B$ associated with organophosphate resistance in mosquitoes of the Culex pipiens complex. Biochem Genet 1981, 19:909-919.

13. Field LM, Williamson MS, Moores GD, Devonshire AL: Cloning and analysis of the esterase genes conferring insecticide resistance in the peachpotato aphid, Myzus persicae (Sulzer). Biochem J 1993, 294:569-574.

14. Ishida Y, Leal WS: Rapid inactivation of a moth pheromone. Proc Natl Acad Sci USA 2005, 102:14075-14079.

15. David JP, Strode C, Vontas J, Nikou D, Vaughan A, Pignatelli PM, Louis C, Hemingway J, Ranson H: The Anopheles gambiae detoxification chip: a highly specific microarray to study metabolicbased insecticide resistance in malaria vectors. Proc Natl Acad Sci USA 2005, 102:4080-4084.

16. Shen B, Dong HQ, Tian HS, Ma L, Li XL, Wu GL: Cytochrome P450 genes expressed in the deltamethrin-susceptible and -resistant strains of Culex pipiens pallus. Pestic Biochem Physiol 2003, 75:19-26.

17. Strode C, Wondji CS, David JP, Hawkes NJ, Lumjuan N, Nelson DR, Drane DR, Karunaratne SH, Hemingway J, Black WC IV, Ranson H: Genomic analysis of detoxification genes in the mosquito Aedes aegypti. Insect Biochem Mol Biol 2008, 38:113-123.

18. Li XC, Schuler MA, Berenbaum MR: Molecular mechanisms of metabolic resistance to synthetic and natural xenobiotics. Annu Rev Entomol 2007, 52:231-253.

19. Puinean AM, Foster SP, Oliphant L, Denholm I, Field LM, Millar NS, Williamson MS, Bass C: Amplification of a cytochrome P450 gene is associated with resistance to neonicotinoid insecticides in the aphid Myzus persicae. PLoS Genet 2010, 6:e1000999.

20. Zhu F, Parthasarathy R, Bai H, Woithe K, Kaussmann M, Nauen R, Harrison DA, Palli SR: A brain-specific cytochrome $\mathrm{P} 450$ responsible for the majority of deltamethrin resistance in the QTC279 strain of Tribolium castaneum. Proc Natl Acad Sci USA 2010, 107:8557-8562.

21. Pedra JH, McIntyre LM, Scharf ME, Pittendrigh BR: Genome-wide transcription profile of field- and laboratory-selected dichlorodiphenyltrichloroethane (DDT)-resistant Drosophila. Proc Natl Acad Sci USA 2004, 101:7034-7039.

22. Ranson H, Rossiter $L$, Ortelli F, Jensen B, Wang X, Roth CW, Collins FH, Hemingway J: Identification of a novel class of insect glutathione S-transferases involved in resistance to DDT in the malaria vector Anopheles gambiae. Biochem J 2001, 359:295-304.

23. Motoyama N, Dauterman WC: The role of nonoxidative metabolism in organophosphorus resistance. J Agric Food Chem 1974, 22:350-356.

24. Sabourault C, Guzov VM, Koener JF, Claudianos C, Plapp FW Jr, Feyereisen R: Overproduction of a P450 that metabolizes diazinon is linked to a loss-of-function in the chromosome 2 ali-esterase (MdalphaE7) gene in resistant house flies. Insect Mol Biol 2001, 10:609-618.

25. Dunkov BC, Mocelin G, Shotkoski F, ffrench-Constant RH, Feyereisen R: The Drosophila cytochrome p450 gene Cyp6a2: structure, chromosomal localisation, heterologous expression and induction by Phenobarbital. DNA Cell Biol 1997, 16:1345-1356.

26. Georghiou GP, Metcalf RL, Gidden FE: Carbamate resistance in mosquitoes: selection of Culex pipiens fatigans Wied. (= Culex quinquefasciatus) for resistance to Baygon. Bull World Health Org 1966, 35:691-708

27. Lawson D, Arensburger P, Atkinson P, Besanksy NJ, Bruggner RV, Butler R, Campbell KS, Christophides GK, Christley S, Dialynas E, Hammond M, Hill CA, Konopinski N, Lobo NF, MacCallum RM, Madey G, Megy K, Meyer J, Redmond S, Severson DW, Stinson E, Topalis P, Birney E, Gelbart WM, Kafatos FC, Louis C, Collins FH: VectorBase: a data resource for invertebrate vector genomics. Nucleic Acids Res 2009, 37:D583-D587. 
28. Ranson H, Claudianos C, Ortelli F, Abgrall C, Hemingway J, Sharakhova MV: Evolution of supergene families associated with insecticide resistance. Science 2002, 298:179-181.

29. Audic S, Claverie JM: The significance of digital gene expression profiles. Genome Res 1997, 7:986-995.

30. Benjamini $Y$, Hochberg $Y$ : Controlling the false discovery rate: a practical and powerful approach to multiple testing. J R Stat Soc Series B Stat Methodol 1995, 57:289-300

31. Beissbarth T, Speed TP: GOstat: find statistically overrepresented gene ontologies within a group of genes. Bioinformatics 2004, 20:1464-1465.

doi:10.1186/1471-2164-13-609

Cite this article as: Yan et al:: Transcriptomic and phylogenetic analysis of Culex pipiens quinquefasciatus for three detoxification gene families. BMC Genomics 2012 13:609.

\section{Submit your next manuscript to BioMed Central and take full advantage of:}

- Convenient online submission

- Thorough peer review

- No space constraints or color figure charges

- Immediate publication on acceptance

- Inclusion in PubMed, CAS, Scopus and Google Scholar

- Research which is freely available for redistribution 